\title{
Editorial
}

\section{Metacaspases are not caspases - always doubt}

\author{
M Enoksson ${ }^{1}$ and GS Salvesen ${ }^{\star, 1}$ \\ Cell Death and Differentiation (2010) 17, 1221; doi:10.1038/cdd.2010.45
}

We read with great interest a recent editorial in $C D D$ by Carmona-Gutierrez et al. with the provocative title of 'Metacaspases are caspases. Doubt no more'. ${ }^{1}$ We agree with the authors that there has been some confusion over the years since the unfortunate naming of the distant caspase orthologs known as 'metacaspases' and 'paracaspases', ${ }^{2}$ and that this confusion has led to misinterpretations in the field of cell death mechanisms. The great philosopher Karl Popper is famous for his attempt to reject the classical observationalist/ inductivist approach to science and to advance empirical falsification in its place: scientists can never prove anything, they can only disprove a thing. Clearly there is always doubt - it would be antiscientific to suggest otherwise - and we suggest that metacaspases have in fact been proven not to be caspases.

Yeast and plants have programmed cell death pathways, but not apoptosis, which is an invention of multicellular animals. ${ }^{3,4}$ Carmona-Gutierrez et al. ${ }^{5}$ use a recent article on plant metacaspase as proof of concept for their statement that metacaspases are caspases. Caspase-3 cleaves human TSN, whereas a plant metacaspase, mcll-Pa, processes recombinant plant TSN. There are however quite striking differences between these observations. For example, mcll-Pa processes recombinant PaTSN into five different fragments - a sign of degradation, whereas human caspase-3 cleaves human TSN at only one site - a sign of the limited proteolytic signaling typical of caspases. ${ }^{6}$ These results do not rule out that metacaspases may be involved in plant cell death and that they might even have a role in plant development. So if we grant that the metacaspase mcll-Pa is involved in a plant cell death pathway, and that this pathway may even be a distant progenitor of apoptosis, does this make metacaspases = caspases? Calpains and cathepsins are involved in necrotic cell death, ${ }^{7}$ but this does not make them caspases. So what is a caspase?

It was the decision of a group of early pioneers to propose the term caspase about 14 years ago, ${ }^{8}$ in a paper containing the important paragraph: 'We propose to use the trivial name 'caspase' as a root for serial names for all family members. The selection of caspase was based on two catalytic properties of these enzymes. The ' $c$ ' is intended to reflect a cysteine protease mechanism, and 'aspase' refers to their ability to cleave after aspartic acid, the most distinctive catalytic feature of this protease family'. The authors of the caspase nomenclature guidelines naively presumed that readers would assume that 'ability to cleave after aspartic acid' actually meant 'prefer to cleave after aspartic acid'. Nevertheless, this specificity, with controversial exceptions ${ }^{9-11}$ has stood the test of time as the keystone of the definition. ${ }^{12}$ Proteases are defined by their catalytic mechanism and substrate specificity. Sure, metcaspases share the same catalytic mechanism as caspases, but what about specificity? The evolutionarily and structurally related clan to which caspases belong includes legumains (specific for Asn), gingipains (specific for Arg and Lys), ${ }^{13,14}$ separase (specific for Arg), ${ }^{15}$ and metacaspases (specific for Arg or Lys). ${ }^{16}$ The very different primary specificity (almost opposite) of caspases from these other groups is not a trivial issue - it is definitive. All of these proteases share a common ancestor, but of course this was neither a metacaspase nor a caspase.

Attempts to create unified themes in biology by comparing similar, yet different, signaling pathways by trying to give them the same attributes cause more confusion than enlightenment. Is this simply a semantic issue? Suppose metacaspases had been called 'metaseparases' - after all they are almost as evolutionarily related as caspases are to metacaspases, and separase shares with metacaspases a primary specificity for Arg. Would we all then be looking for roles of metacaspases (metaseparases?) in anaphase progression? Metacaspases are not caspases by any useful definition, the disproof is evident. No doubt.

\section{Conflict of interest}

The authors declare no conflict of interest.

\footnotetext{
1. Carmona-Gutierrez D et al. Cell Death Differ 2010; 17: 377-378.

2. Uren AG et al. Mol Cell 2000; 6: 961-967.

3. Hardwick JM et al. Dev Cell 2004; 7: 630-632.

4. Zmasek CM et al. Genome Biol 2007; 8: R226.

5. Sundstrom JF et al. Nat Cell Biol 2009; 11: 1347-1354.

6. Timmer JC, Salvesen GS. Cell Death Differ 2007; 14: 66-72.

7. Syntichaki $P$ et al. Nature 2002; 419: 939-944.

8. Alnemri ES et al. Cell 1996; 87: p171.

9. Krippner-Heidenreich A et al. Biochem J 2001; 358: 705-715.

10. Hawkins CJ et al. J Biol Chem 2000; 275: 27084-27093.

11. Snipas SJ et al. Cell Death Differ 2008; 15: 938-945.

12. Thornberry NA et al. J Biol Chem 1997; 272: 17907-17911.

13. Aravind L, Koonin EV. Proteins 2002; 46: 355-367.

14. Barrett AJ, Rawlings ND. Biol Chem 2001; 382: 727-733.

15. Uhlmann F et al. Cell 2000; 103: 375-386.

16. Vercammen D et al. J Biol Chem 2004; 279: 45329-45336.
}

\footnotetext{
${ }^{1}$ Program in Apoptosis and Cell Death Research, Sanford-Burnham Medical Research Institute, La Jolla, CA, USA

*Correspondence: GS Salvesen, Program in Apoptosis and Cell Death Research, Sanford-Burnham Medical Research Institute, 10901 North Torrey Pines Road, La Jolla, CA 92037, USA. Tel: +1 858646 3114; Fax: +1 858713 6274; E-mail: gsalvesen@ burnham.org
} 